\title{
Empagliflozin reduziert das Risiko für Mortalität sowie makro- und mikrovaskuläre Komplikationen bei Patienten mit Typ-2-Diabetes und bestehender kardiovaskulärer Erkrankung
}

\author{
Empagliflozin Reduces the Risk of Mortality, Macro- and \\ Microvascular Events in Patients with Type 2 Diabetes \\ and Established Cardiovascular Disease
}

Autoren

Jochen Seufert ${ }^{1}$, Jan-Christoph Galle ${ }^{2}$, Martina Manning ${ }^{3}$, Volkmar Schmid ${ }^{4}$, Michael Lehrke ${ }^{5}$

Institute

1 Abteilung Endokrinologie und Diabetologie, Klinik für Innere Medizin II, Universitätsklinikum Freiburg, Medizinische Fakultät, Albert-Ludwigs-Universität Freiburg

2 Klinik für Nephrologie und Dialyseverfahren, Märkische Kliniken GmbH Klinikum Lüdenscheid, Germany

3 Medizinische Abteilung, Lilly Deutschland GmbH, Bad Homburg, Germany

4 Medical Affairs Deutschland, Boehringer Ingelheim Pharma GmbH \& Co. KG, Ingelheim, Germany

5 Medizinische Klinik I, Klinik für Kardiologie, Pneumologie, Angiologie und Internistische Intensivmedizin, Universitätsklinikum Aachen, Germany

Schlüsselwörter

Empagliflozin, SGLT-2-Inhibitor, Diabetes mellitus, Mortalität, Kardiovaskuläre Endpunktstudie

Key words

cardiovascular outcome trial, empagliflozin, SGLT-2 inhibitor, diabetes mellitus, mortality

eingereicht 13.02 .2017

akzeptiert 30.05.2017

Bibliografie

DOI https://doi.org/10.1055/s-0043-112658 |

Diabetologie 2017; 12: 294-306 @ Georg Thieme Verlag KG, Stuttgart · New York, ISSN 1861-9002

Korrespondenzadresse

Dr. Volkmar Schmid

Medical Affairs Deutschland, Boehringer Ingelheim Pharma GmbH \& Co. KG, Binger Straße 173, 55216 Ingelheim,

Germany

Tel.: ++49/6132/77142057

volkmar.schmid@boehringer-ingelheim.com

\section{ZUSAMMENFASSUNG}

Patienten mit Typ-2-Diabetes mellitus haben auch bei optimierter Lipidsenkung, Blutdruckkontrolle und Blutzuckereinstellung ein erhöhtes Risiko für kardiovaskuläre Ereignisse. Zur Abbildung der kardiovaskulären Sicherheit neu zugelassener Antidiabetika verlangen die Arzneimittelbehörden FDA und EMA den Nachweis für Nichtunterlegenheit bezüglich kardiovaskulärer Sicherheit gegenüber Placebo in Endpunktstudien. Während für verschiedene neuere Antidiabetika bisher die Nichtunterlegenheit in kardiovaskulären Sicherheitsstudien gezeigt wurde, konnte mit Empagliflozin erstmals in der EMPA-REG OUTCOME ${ }^{\circledR}$-Studie und nachfolgend mit Liraglutid in der LEADER-Studie eine präspezifizierte kardiovaskuläre Überlegenheit im Vergleich zu Placebo demonstriert werden; auch mit Semaglutid wurde in der SUSTAIN-6-Studie eine statistisch signifikante Reduktion kardiovaskulärer Ereignisse erreicht. Die Gabe von Empagliflozin, einem Hemmer des Natrium-Glukose-Cotransporters 2 (SGLT-2), zusätzlich zur antidiabetischen und kardiovaskulären Standardtherapie führte zu einer signifikanten relativen Risikoreduktion des primären kombinierten Endpunkts aus kardiovaskulärem Tod, nicht tödlichem Myokardinfarkt oder nicht tödlichem Schlaganfall um $14 \%$ gegenüber Placebo. Diese Risikoreduktion wurde maßgeblich durch die Senkung der kardiovaskulären Mortalität um $38 \%$ erreicht. Zudem zeigten sich gegenüber Placebo relative Risikoreduktionen von $35 \%$ bzw. 39 \% für stationäre Aufnahmen wegen Herzinsuffizienz bzw. für das Neuauftreten oder die Verschlechterung einer Nephropathie. Empagliflozin reduzierte die Gesamtmortalität relativ um $32 \%$. Welche Mechanismen den nachgewiesenen Risikoreduktionen zugrunde liegen, ist Gegenstand weiterer Untersuchungen. Diskutiert werden vor allem eine Entlastung des Herzens durch Ausscheidung von überschüssigem Natrium, Glukose und Wasser, hämodynamische Effekte an der Niere über eine Normalisierung des tubulo-glomerulären Feedbacks oder auch eine durch Ketonkörper energetisch günstigere Substratnutzung im vorgeschädigten Herzen. Empagliflozin war, mit Ausnahme einer erhöhten 
Rate an Genitalinfektionen, die generell mit dem Wirkmechanismus von SGLT-2-Hemmern assoziiert sind, gut verträglich.

\section{ABSTRACT}

Patients with type 2 diabetes mellitus face an increased risk of cardiovascular events, even under conditions of optimal lipid reduction, blood pressure and blood glucose control. To ensure cardiovascular safety of novel antidiabetic drugs, FDA and EMA regulations require evidence of non-inferiority compared to placebo in cardiovascular outcome studies for approval. Previously, cardiovascular safety of various newer antidiabetic drugs was observed in dedicated non-inferiority trials. In contrast, the EMPA-REG OUTCOME ${ }^{\circledR}$ study was the first trial to demonstrate for empagliflozin, followed by the LEADER trial for liraglutide, prespecified cardiovascular superiority of an individual antidiabetic medication versus placebo. Subsequently, semaglutide showed a significant reduction in cardiovascular events in the SUSTAIN 6 trial. Administration of empagliflozin, a sodium glucose-linked cotransporter (SGLT-2) inhibitor, in addition to antidiabetic and cardiovascu- lar standard of care medication led to a significant relative risk reduction by $14 \%$ of the primary composite endpoint composed of cardiovascular death, non-fatal myocardial infarction, or non-fatal stroke as compared to placebo. This overall risk reduction was mainly driven by a significant $38 \%$ reduction in cardiovascular death. Furthermore, empagliflozin reduced the relative risk of hospitalisations for heart failure, or incident or worsening nephropathy by $35 \%$ and $39 \%$, respectively. Empagliflozin reduced the relative risk of all-cause mortality by $32 \%$. The underlying mechanisms for the observed risk reductions are not yet completely understood. Specifically, relieving the heart from excess glucose, sodium and water, effects on renal hemodynamics and restored tubuloglomerular feedback as well as a substrate shift towards ketone bodies resulting in better energy consumption in the diabetic heart are considered. Empagliflozin was well tolerated but associated with an increased incidence of genital infections, which are generally associated with the mode of action of SGLT-2 inhibitors.

\section{Einleitung}

Patienten mit Typ-2-Diabetes mellitus (T2DM) besitzen ein zweibis vierfach höheres Risiko, eine kardiovaskuläre Erkrankung zu entwickeln, als Menschen ohne T2DM [1, 2]. Die koronare Herzkrankheit zeigt dabei die höchste Prävalenz unter den kardiovaskulären Erkrankungen bei Menschen mit T2DM, gefolgt von Schlaganfall und peripherer arterieller Verschlusskrankheit. Darüber hinaus wurde das Risiko der Entwicklung einer Herzinsuffizienz, die wesentlich die Prognose der Patienten mit T2DM verschlechtert, lange unterschätzt [3]. Bei Männern und Frauen über 50 Jahren mit T2DM und einer kardiovaskulären Vorerkrankung ist die Lebenserwartung im Durchschnitt um 7,5 bzw. 8,2 Jahre verkürzt, wobei mehr als die Hälfte der Todesfälle durch ein kardiovaskuläres Ereignis verursacht werden [4, 5]. Demzufolge steht die Senkung der makro- und mikrovaskulären Folgeerkrankungen durch eine adäquate Therapie des T2DM im Mittelpunkt nationaler und internationaler Leitlinien und Behandlungsempfehlungen [6, 7]. Ältere Endpunktstudien haben gezeigt, dass das Risiko mikrovaskulärer Komplikationen durch eine gute Blutzuckerkontrolle reduziert werden kann [8, 9]. Trotzdem ist Diabetes mellitus in vielen Teilen der Welt nach wie vor die häufigste Ursache für die Entwicklung einer terminalen Niereninsuffizienz [10 12]. Bis zu $40 \%$ der Patienten mit T2DM sind früher oder später von einer Niereninsuffizienz betroffen, wobei die interindividuelle Varianz bis zum Eintreten der ersten Krankheitszeichen sehr groß ist (wenige Jahre bis Jahrzehnte) [11-13]. Den Nachweis, dass durch eine intensive Blutzuckertherapie eine Reduktion makrovaskulärer Ereignisse erreicht wird, blieben ältere Outcomestudien hingegen im Wesentlichen schuldig, da die Blutzuckersenkung wenig Auswirkungen auf das kardiovaskuläre Risiko im Verlauf der Studienbeobachtung hatte. Mehrjährige Nachbeobachtungen nach Abschluss der Studien konnten teilweise eine signifikante Senkung kardiovaskulärer Ereignisse durch eine frühe intensive Blutzuckersenkung nachweisen [14-16]. Blutzuckerkontrolle per se scheint sich erst langfristig positiv auf das kardiovaskuläre Risiko auszuwirken. Demzufolge liegt die Inzidenzrate von Myokardinfarkt, Schlaganfall und kardiovaskulärer Mortalität bei Patienten mit T2DM deutlich höher als in der Allgemeinbevölkerung. Aufgrund der stetig steigenden Prävalenz von T2DM ist damit eine weitere Zunahme kardiovaskulärer Komplikationen zu erwarten [14].

\section{Studien zur Beurteilung der kardiovaskulä- ren Sicherheit}

Während frühere klinische Studien kaum einen Vorteil der Blutzuckerkontrolle hinsichtlich makrovaskulärer Ereignisse bei Patienten mit T2DM zeigen konnten und in der ACCORD-Studie sogar eine Übersterblichkeit unter intensiver Blutzuckertherapie zu verzeichnen war [17 - 19], rückte zunehmend der Aspekt der kardiovaskulären Sicherheit einer antidiabetischen Therapie auf der Basis von Einzelsubstanzen in den Vordergrund. Nachdem in einer inzwischen widerlegten Metaanalyse ein erhöhtes Risiko für Myokardinfarkt und Tod durch kardiovaskuläre Ereignisse für Rosiglitazon beschrieben wurde [20], ist seit 2008 durch die Zulassungsbehörden der Nachweis einer langfristigen kardiovaskulären Sicherheit vor und nach Zulassung neuer Antidiabetika vorgegeben [21]. In der EMPA-REG OUTCOME ${ }^{\circledR}$-Studie mit dem Inhibitor des Natrium-Glukose-Cotransporters 2 (SGLT-2) Empagliflozin konnte erstmals eine Überlegenheit im primären Endpunkt MACE-3 zugunsten eines einzelnen Wirkstoffs gezeigt werden [22]. Auch in der LEADER-Studie konnte nachfolgend eine Überlegenheit des einmal täglich zu injizierenden GLP-1-Rezeptoragonisten Liraglutid beim Auftreten des primären Endpunkts 
MACE-3 nachgewiesen werden [23]. Für einen weiteren GLP-1-Rezeptoragonisten, das einmal wöchentlich zu injizierende Semaglutid, wurde im Rahmen einer Phase-3-Zulassungsstudie ebenfalls ein Vorteil beim Auftreten von MACE-3-Ereignissen gegenüber Placebo demonstriert [24].

\section{EMPA-REG OUTCOME ${ }^{\circledR}$-Studie}

Empagliflozin zeigte bereits im Phase-3-Studienprogamm, dass die Substanz den $\mathrm{HbA}_{1 c}$, das Körpergewicht und den Blutdruck klinisch relevant senkt und, abgesehen von Genitalinfektionen, die durch den Wirkmechanismus (Induktion einer Glukosurie) bedingt sind, ein positives Verträglichkeitsprofil aufweist [25]. Die Untersuchung makro- und mikrovaskulärer Endpunkte bei Patienten mit T2DM und bestehender kardiovaskulärer Erkrankung in der EMPA-REG OUTCOME ${ }^{\circledR}$-Studie erfolgte nach regulatorischen Vorgaben.

\section{Studiendesign}

EMPA-REG OUTCOME ${ }^{\circledR}$ (ClinicalTrials.gov Nummer: NCT01 131676 ) ist eine internationale, randomisierte, doppelblinde Studie mit drei Behandlungsarmen (Empagliflozin $10 \mathrm{mg}$ oder $25 \mathrm{mg}$ oder Placebo, jeweils zusätzlich zur antidiabetischen und kardiovaskulären Standardtherapie; Abb. 1), die in 42 Ländern durchgeführt wurde. Eingeschlossen wurden Patienten mit einem $\mathrm{HbA}_{1 c}$ von 7 - 10\% und hohem kardiovaskulärem Risiko aufgrund vorbestehender Herz-Kreislauf-Erkrankungen. Die bisherige antidiabetische und kardiovaskuläre Standardtherapie (Antidiabetika, Antihypertensiva, Lipidsenker und Thrombozytenaggregationshemmer) wurde beibehalten und sollte im Studienverlauf kontinuierlich und leitliniengerecht angepasst werden, um möglichst in allen Studienarmen vergleichbare Zielwerte zu erreichen. Der primäre kombinierte Endpunkt war die Zeit bis zum ersten Auftreten eines MACE-3-Ereignisses (kardiovaskulärer Tod, nicht tödlicher Myokardinfarkt oder nicht tödlicher Schlaganfall). Die Studie war ereignisgesteuert und wurde im Hinblick auf die statistische Aussagekraft so lange durchgeführt, bis bei zumindest 691 Patienten ein MACE-3-Ereignis eingetreten war. Der wichtigste sekundäre Endpunkt war MACE-4 (MACE-3 plus Hospitalisierung aufgrund von instabiler Angina pectoris). Weitere kardiovaskuläre Endpunkte waren unter anderem die Einzelkomponenten von MACE-4 sowie Hospitalisierung aufgrund von Herzinsuffizienz, Gesamtmortalität, transiente ischämische Attacke (TIA), neu auftretende Albuminurie und neue oder sich verschlechternde Nephropathie (kombinierter Endpunkt aus Progression zur Makroalbuminurie, Verdopplung des Serum-Kreatinin-Werts bei gleichzeitiger eGFR [geschätzte glomeruläre Filtrationsrate] $\leq 45 \mathrm{ml} / \mathrm{min}$, Einleitung einer Nierenersatztherapie oder Tod durch Nierenerkrankung) [26]. Zudem wurde die Wirkung von Empagliflozin auf $\mathrm{HbA}_{1 \mathrm{c}}$, Nüchternblutzucker, Körpergewicht, Bauchumfang, Blutdruck, Pulsrate, Lipide, Harnsäure und eGFR zu vorab definierten Zeitpunkten während des Studienverlaufs gemessen ( $\boldsymbol{A}$ Abb. 1). Die Dokumentation unerwünschter Ereignisse erfolgte über die gesamte Studiendauer. Im Studienverlauf auftretende Todesfälle sowie andere zu MACE gehörende Ereignisse wurden von mehreren verblindeten, externen, klinischen Event-Komitees bewertet [22].

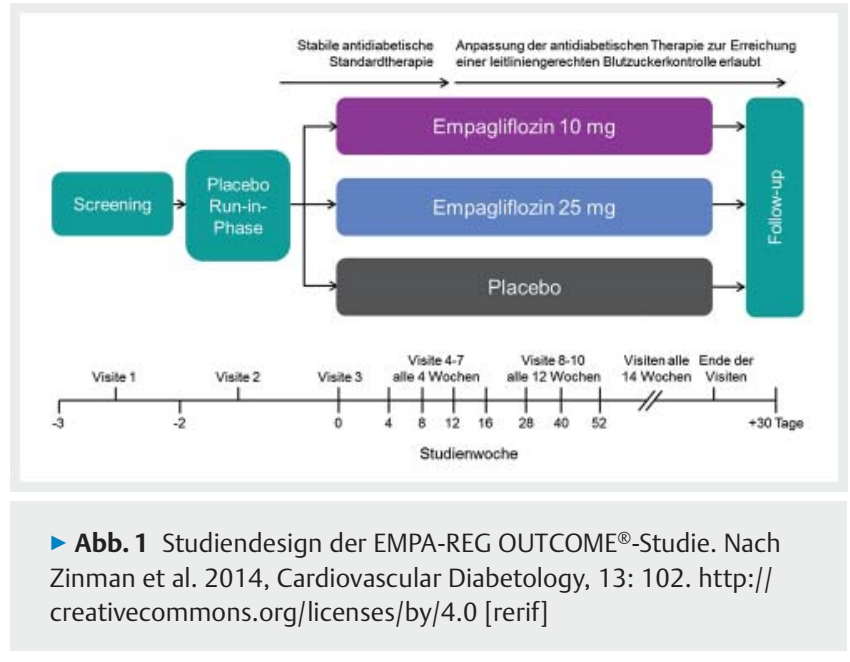

Die Studie war von Beginn an statistisch darauf ausgelegt, in hierarchischer Testung zunächst Nichtunterlegenheit und dann Überlegenheit zeigen zu können (Empagliflozin vs. Placebo für MACE-3 und MACE-4). Für die primäre Analyse wurden die Daten der beiden Empagliflozin-Gruppen (10 mg und 25 mg) gepoolt. Darüber hinaus wurden verschiedene Subgruppen analysiert (Alter zu Studienbeginn, Geschlecht, Rasse, Ethnizität, Region, $\mathrm{HbA}_{1 \mathrm{c}}$, BMI, Blutdruck, eGFR, Albumin-Kreatinin-Ratio, kardiovaskuläre Risikofaktoren, antidiabetische Medikation, Anwendung von Statinen, Antihypertonika, Antikoagulanzien) [26].

\section{Patienten-Charakteristika}

Insgesamt wurden 7042 Patienten in 592 Studienzentren eingeschlossen. Nach der zweiwöchigen Run-in-Phase wurden $7034 \mathrm{~Pa}$ tienten (72\% männlich, durchschnittliches Alter 63,1 Jahre) auf die 3 Behandlungsarme randomisiert. Insgesamt flossen die Daten von 7020 Patienten in die Primäranalyse ein. Die mediane Beobachtungsdauer betrug 3,1 Jahre. Insgesamt beendeten 97,0 \% der Patienten die Studie, und zu Studienende war der Vitalstatus für 99,2\% der Patienten bekannt [22]. Die Baseline-Charakteristika waren in den drei Behandlungsgruppen vergleichbar ( $\triangleright$ Tab. 1). Der mittlere $\mathrm{HbA}_{1 \mathrm{c}}$-Wert zu Studienbeginn betrug $8,1 \%$. Fast alle Patienten ( $99 \%$ ) hatten eine kardiovaskuläre Vorerkrankung (47\% Myokardinfarkt, 23 \% Schlaganfall). Etwa ein Zehntel der Patienten litt unter Herzinsuffizienz. Die Mehrheit der Patienten wies eine eingeschränkte Nierenfunktion im Sinne einer eGFR (gemäß CKD-EPI-Formel) unter $90 \mathrm{ml} / \mathrm{min} / 1,73 \mathrm{~m}^{2}$ auf (52\% CKD-Stadium 2, $26 \%$ CKD-Stadium 3). Eine Albuminurie war zu Studienbeginn bei $40 \%$ der Patienten bekannt. Insgesamt wies zu Studienbeginn ein großer Anteil der Patienten eine gute Versorgung mit Antidiabetika (Metformin, ca. 74\%; Insulin, ca. $48 \%$ ), Lipidsenkern (ca. 80\%), Antihypertensiva (ca. $95 \%$ ) sowie Antikoagulanzien/Thrombozytenaggregationshemmern (ca. $89 \%$ ) auf ( Tab. 1) [22].

\section{Wirksamkeit}

Die nachfolgend beschriebenen Ergebnisse werden für die gepoolten Daten beider Empagliflozin-Dosierungen (10 mg und $25 \mathrm{mg}$ ) dargestellt. 
- Tab. 1 Baseline-Charakteristika.

\begin{tabular}{|c|c|c|c|}
\hline Parameter & $\begin{array}{l}\text { Placebo } \\
(\mathrm{N}=2333)\end{array}$ & $\begin{array}{l}\text { Empagliflozin } 10 \mathrm{mg} \\
\qquad(\mathrm{N}=2345)\end{array}$ & $\begin{array}{l}\text { Empagliflozin } 25 \mathrm{mg} \\
\qquad(\mathrm{N}=2342)\end{array}$ \\
\hline Alter, Jahre (MW \pm SD) & $63,2 \pm 8,8$ & $63,0 \pm 8,6$ & $63,2 \pm 8,6$ \\
\hline Männlich, n (\%) & $1680(72,0 \%)$ & $1653(70,5 \%)$ & $1683(71,9 \%)$ \\
\hline $\mathrm{HbA}_{1 \mathrm{c}}, \%$ & $8,08 \pm 0,84$ & $8,07 \pm 0,86$ & $8,06 \pm 0,84$ \\
\hline \multicolumn{4}{|l|}{ Anzahl Jahre seit der T2DM-Diagnose (MW $\pm S D)$} \\
\hline$\cdot \leq 5$ & $423 \pm 18,1$ & $406 \pm 17,3$ & $434 \pm 18,5$ \\
\hline . $>5-10$ & $571 \pm 24,5$ & $585 \pm 24,9$ & $590 \pm 25,2$ \\
\hline . $>10$ & $1339 \pm 57,4$ & $1354 \pm 57,7$ & $1318 \pm 56,3$ \\
\hline \multicolumn{4}{|l|}{ Antidiabetische Medikation, $\mathrm{n}(\%)$} \\
\hline - Metformin & $1734(74,3 \%)$ & $1729(73,7 \%)$ & $1730(73,9 \%)$ \\
\hline - Sulfonylharnstoff & $992(42,5 \%)$ & $985(42,0 \%)$ & $1029(43,9 \%)$ \\
\hline - DPP-4-Inhibitor & $267(11,4 \%)$ & $282(12,0 \%)$ & $247(10,5 \%)$ \\
\hline - Thiazolidindione & $101(4,3 \%)$ & $96(4,1 \%)$ & $102(4,4 \%)$ \\
\hline - Insulin & $1135(48,6 \%)$ & $1132(48,3 \%)$ & $1120(47,8 \%)$ \\
\hline - Insulin-Tagesdosis, U (MW \pm SD) & $65 \pm 50,6$ & $65 \pm 47,9$ & $66 \pm 48,9$ \\
\hline \multicolumn{4}{|l|}{ Kardiovaskuläre Risikofaktoren } \\
\hline $\mathrm{BMI}, \mathrm{kg} / \mathrm{m}^{2}(\mathrm{MW} \pm \mathrm{SD})$ & $30,7 \pm 5,2$ & $30,6 \pm 5,2$ & $30,6 \pm 5,3$ \\
\hline Systolischer Blutdruck, mmHg (MW \pm SD) & $135,8 \pm 17,2$ & $134,9 \pm 16,8$ & $135,6 \pm 17,0$ \\
\hline Diastolischer Blutdruck, $\mathrm{mmHg}(\mathrm{MW} \pm \mathrm{SD})$ & $76,8 \pm 10,1$ & $76,6 \pm 9,8$ & $76,6 \pm 9,7$ \\
\hline LDL-Cholesterin, mg/dl (MW $\pm \mathrm{SD})$ & $84,9 \pm 35,3$ & $86,3 \pm 36,7$ & $85,5 \pm 35,2$ \\
\hline HDL-Cholesterin, mg/dl (MW \pm SD) & $44,0 \pm 11,3$ & $44,7 \pm 12,0$ & $44,5 \pm 11,8$ \\
\hline \multicolumn{4}{|l|}{ eGFR, n (\%) } \\
\hline . $\geq 90 \mathrm{ml} / \mathrm{min} / 1,73 \mathrm{~m}^{2}$ & $488(20,9 \%)$ & $519(22,1 \%)$ & $531(22,7 \%)$ \\
\hline - $60-<90 \mathrm{ml} / \mathrm{min} / 1,73 \mathrm{~m}^{2}$ & $1238(53,1 \%)$ & $1221(52,1 \%)$ & $1202(51,3 \%)$ \\
\hline . $<60 \mathrm{ml} / \mathrm{min} / 1,73 \mathrm{~m}^{2}$ & $607(26,0 \%)$ & $605(25,8 \%)$ & $607(25,9 \%)$ \\
\hline \multicolumn{4}{|l|}{ Kardiovaskuläre Erkrankung } \\
\hline 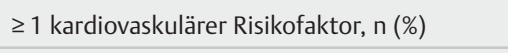 & $2307(98,9 \%)$ & $2333(99,5 \%)$ & $2324(99,2 \%)$ \\
\hline - Koronare Herzkrankheit & $1763(75,6 \%)$ & $1782(76,0 \%)$ & $1763(75,3 \%)$ \\
\hline - Myokardinfarkt in der Anamnese & $1083(46,4 \%)$ & $1107(47,2 \%)$ & $1083(46,2 \%)$ \\
\hline - Koronararterien-Bypass & $563(24,1 \%)$ & $594(25,3 \%)$ & $581(24,8 \%)$ \\
\hline - Schlaganfall in der Anamnese & $553(23,7 \%)$ & $535(22,8 \%)$ & $549(23,4 \%)$ \\
\hline - Periphere arterielle Verschlusskrankheit & $479(20,5 \%)$ & $465(19,8 \%)$ & $517(22,1 \%)$ \\
\hline Herzinsuffizienz & $244(10,5 \%)$ & $240(10,2 \%)$ & $222(9,5 \%)$ \\
\hline \multicolumn{4}{|l|}{ Kardiovaskuläre Medikation } \\
\hline Blutdrucksenker, n (\%) & $2221(95,2 \%)$ & $2227(95,0 \%)$ & $2219(94,7 \%)$ \\
\hline - ACE-Hemmer/ARB & $1868(80,1 \%)$ & $1896(80,9 \%)$ & $1902(81,2 \%)$ \\
\hline - Betablocker & $1498(64,2 \%)$ & $1530(65,2 \%)$ & $1526(65,2 \%)$ \\
\hline - Diuretika & $988(42,3 \%)$ & $1036(44,2 \%)$ & $1011(43,2 \%)$ \\
\hline - Calciumkanal-Hemmer & $788(33,8 \%)$ & $781(33,3 \%)$ & $748(31,9 \%)$ \\
\hline - Mineralocorticoid-Rezeptor-Antagonisten & $136(5,8 \%)$ & $157(6,7 \%)$ & $148(6,3 \%)$ \\
\hline - Andere & $210(9,0 \%)$ & $209(8,9 \%)$ & $201(8,6 \%)$ \\
\hline
\end{tabular}


- Tab. 1 (Fortsetzung)

\begin{tabular}{|l|c|c|c|}
\hline Parameter & $\begin{array}{c}\text { Placebo } \\
(\mathbf{N}=\mathbf{2 3 3 3})\end{array}$ & $\begin{array}{c}\text { Empagliflozin 10 mg } \\
\mathbf{( N = 2 3 4 5 )}\end{array}$ & $\begin{array}{c}\text { Empagliflozin 25 mg } \\
\mathbf{( N = 2 3 4 2 )}\end{array}$ \\
\hline Lipidsenker, $\mathbf{n}(\%)$ & $1864(79,9 \%)$ & $1926(82,1 \%)$ & $1894(80,9 \%)$ \\
\hline - Statine & $1773(76,0 \%)$ & $1827(77,9 \%)$ & $1803(77,0 \%)$ \\
\hline - Fibrate & $199(8,5 \%)$ & $214(9,1 \%)$ & $217(9,3 \%)$ \\
\hline - Ezetimib & $81(3,5 \%)$ & $95(4,1 \%)$ & $94(4,0 \%)$ \\
\hline - Andere & $210(9,0 \%)$ & $228(9,7 \%)$ & $228(9,7 \%)$ \\
\hline Antikoagulanzien und Thrombozytenaggregations- & $2090(89,6 \%)$ & $2098(89,5 \%)$ & $2064(88,1 \%)$ \\
\hline hemmer & $1927(82,6 \%)$ & $1939(82,7 \%)$ & $1937(82,7 \%)$ \\
\hline - Acetylsalicylsäure & $249(10,7 \%)$ & $253(10,8 \%)$ & $241(10,3 \%)$ \\
\hline - Clopidogrel & $156(6,7 \%)$ & $141(6,0 \%)$ & $125(5,3 \%)$ \\
\hline
\end{tabular}

Die EMPA-REG OUTCOME ${ }^{\circledR}$-Studie war darauf ausgelegt, das Auftreten klinisch relevanter kardiovaskulärer Ereignisse zu untersuchen. Eine effektive Blutzuckerkontrolle durch Empagliflozin in der Mono- oder Kombinationstherapie war bereits durch die Zulassungsstudien belegt [25, 27 - 29]. In der EMPA-REG OUT$\mathrm{COME}^{\circledR}$-Studie war nach 206 Wochen unter Empagliflozin eine geringfügig stärkere mittlere $\mathrm{HbA}_{1 \mathrm{c}}$-Senkung als in der PlaceboGruppe vorhanden (7,81\% versus $8,16 \%)$. Im Vergleich zu Placebo war Empagliflozin im Studienverlauf mit einer leichten Verringerung des Körpergewichts, des Bauchumfangs, des Harnsäurewerts sowie des systolischen und diastolischen Blutdrucks assoziiert. Es wurde keine Erhöhung der Herzfrequenz und geringfügige Zunahmen an LDL- und HDL-Cholesterin beobachtet [22].

\section{Makrovaskuläre Endpunkte}

MACE-3-Ereignisse waren unter Empagliflozin signifikant seltener als unter Placebo (Hazard Ratio [HR] 0,86 (95,02\% KI: 0,74; 0,99), was einer 14-\%igen relativen Risikoreduktion (RRR) und einer signifikanten Überlegenheit gegenüber Placebo entspricht $(p=0,04)$ ( $\vee$ Abb. 2a). Der Unterschied bei MACE-3 wurde getrieben durch eine Reduktion kardiovaskulär bedingter Todesfälle (HR 0,62; $95 \%$ KI: 0,$49 ; 0,77 ; p<0,001$ ) ( $\triangleright$ Abb. 2b), wobei in allen sechs Unterkategorien von kardiovaskulärem Tod der positive Effekt von Empagliflozin vorhanden war, u. a. in den beiden Kategorien tödlicher Myokardinfarkt und tödlicher Schlaganfall [22]. Die Behandlung mit Empagliflozin hatte keinen signifikanten Einfluss auf die Häufigkeit der MACE-3-Komponenten nicht tödlicher Myokardinfarkt (HR 0,87; $95 \% \mathrm{KI}: 0,70 ; 1,09 ; \mathrm{p}=0,22$ ) und Schlaganfall (HR 1,24; 95 \% Kl: 0,92; 1,67; p =0,16). Hinsichtlich MACE-4 zeigte Empagliflozin keine signifikante Überlegenheit im Vergleich zu Placebo (HR 0,89; $95 \% \mathrm{KI}$ : 0,78; 1,01; p=0,08), was auf die durch Empagliflozin unbeeinflusste Häufigkeit von Hospitalisierungen wegen Angina pectoris zurückzuführen ist (HR 0,99; 95 \% KI: 0,74; $1,34 ; p=0,97)[22]$.

Unter Empagliflozin war das als sekundärer Endpunkt präspezifizierte relative Risiko für Krankenhauseinweisungen wegen Herzinsuffizienz gegenüber Placebo signifikant reduziert (HR 0,65; 95 \%

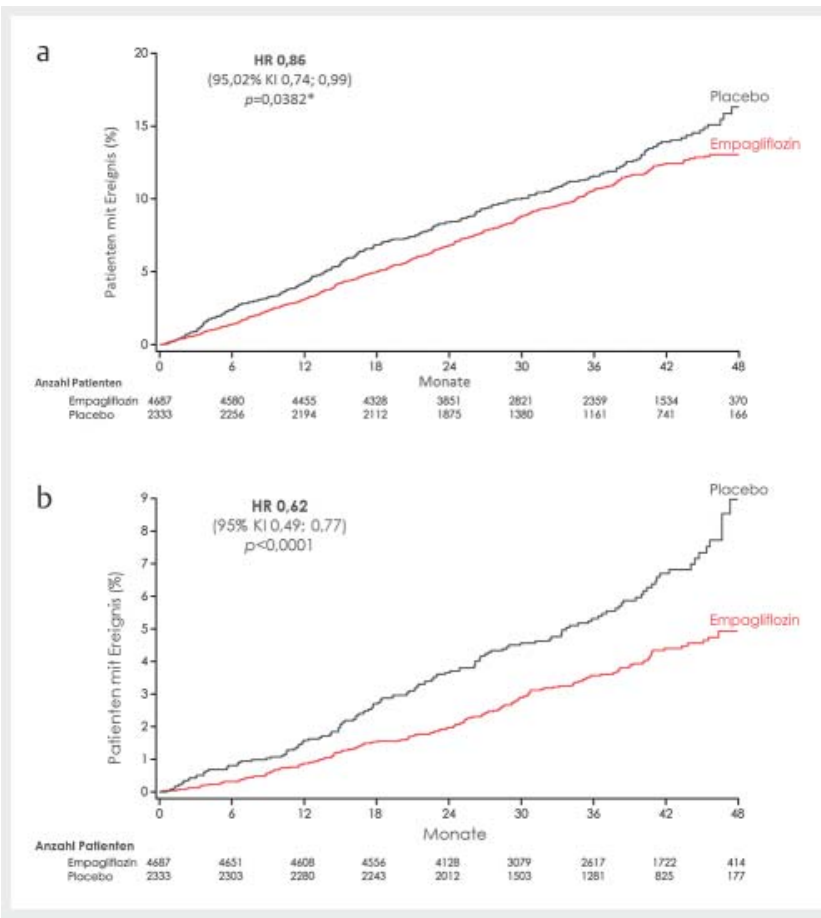

- Abb. 2 Erreichen des primären Endpunkts MACE-3 a und Auftreten kardiovaskulärer Todesfälle b (Nach Zinman et al. 2015, N Engl J Med 373:2117-2128).

KI: 0,50; 0,85; $p=0,002$ ) ( Abb. 3a). Von dieser Risikoreduktion profitierten Patienten unabhängig davon, ob bei ihnen zu Studienbeginn eine Herzinsuffizienz vorlag oder nicht [30]. Krankenhauseinweisungen aufgrund von Herzinsuffizienz oder kardiovaskulärer Tod traten in der Empagliflozin-Gruppe signifikant seltener als in der Placebo-Gruppe auf (5,7\% vs. 8,5\%; HR 0,66; $95 \%$ KI: 0,55; $0,79 ; \mathrm{p}<0,001)$. Der Vorteil von Empagliflozin gegenüber Placebo hinsichtlich kardiovaskulärer Todesfälle wurde in allen Subgruppen und in beiden Empagliflozin-Behandlungsarmen beobachtet. Beim primären Endpunkt und bei kardiovaskulärem Tod lagen die Punkt- 


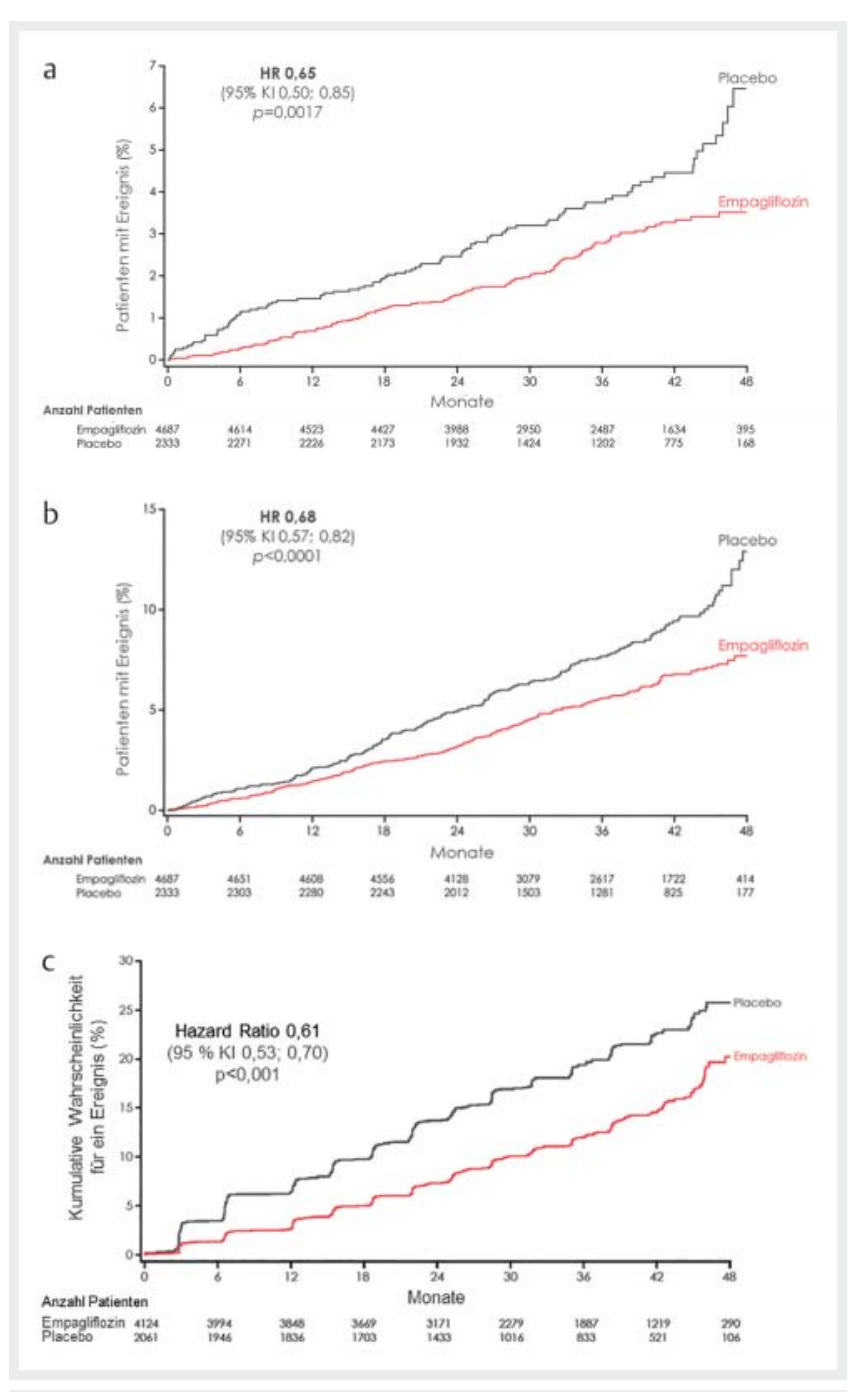

- Abb. 3 Hospitalisierung aufgrund von Herzinsuffizienz a, Gesamtmortalität b und neue oder sich verschlechternde Nephropathie $\mathbf{c}$ (a und b nach Zinman et al. 2015, N Engl J Med 373:2117 2128; c nach Wanner et al. 2016, N Engl J Med 375:323 - 334).

schätzer bei den Subgruppenanalysen nahezu durchweg unter 1 . Dies weist darauf hin, dass die positiven Ergebnisse unter Empagliflozin für ein breites Patientenkollektiv mit bestehender kardiovaskulärer Erkrankung Gültigkeit haben [22].

\section{Gesamtmortalität}

Das relative Risiko für Gesamtmortalität war in der EmpagliflozinGruppe signifikant um $32 \%$ niedriger als in der Placebo-Gruppe (HR 0,68; $95 \%$ Kl: 0,57; 0,82; p<0,0001) ( Abb. 3b) [22].

\section{Renale Endpunkte}

Im Vergleich zu Placebo wurde unter Empagliflozin das Neuauftreten oder eine Verschlechterung einer Nephropathie um $39 \%$ (HR 0,61; 95 \% KI: 0,53; 0,70; p<0,001) ( Abb. 3c) sowie die Progression zur Makroalbuminurie um 38 \% (HR 0,62; 95 \% KI: 0,54; $0,72 ; p<0,001)$ reduziert [31]. Eine noch deutlichere Reduktion um $46 \%$ gegenüber Placebo wurde beim kombinierten Endpunkt

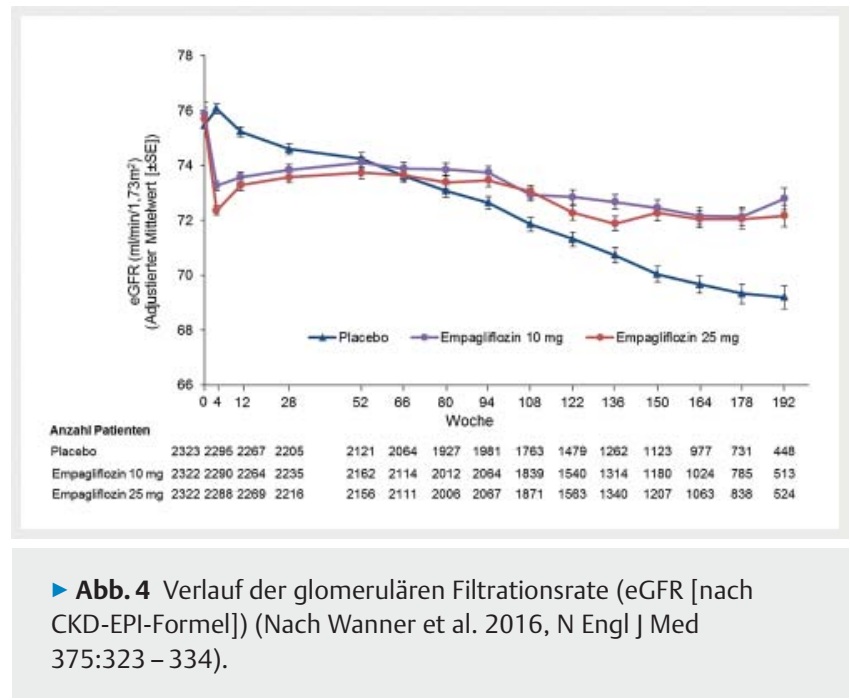

aus Verdopplung der Kreatininkonzentration im Serum, Einleitung einer Nierenersatztherapie oder Tod infolge Nierenerkrankung festgestellt (HR 0,54; $95 \% \mathrm{KI}$ : 0,40; 0,75; p<0,001). In Übereinstimmung traten auch die einzelnen renalen Ereignisse einer Verdopplung der Kreatininkonzentration im Serum (HR 0,56; $95 \% \mathrm{KI}$ : 0,39; 0,79; $p<0,001)$ und der Einleitung einer Nierenersatztherapie (HR 0,45; $95 \% \mathrm{KI}: 0,21 ; 0,97 ; \mathrm{p}=0,04$ ) in der EmpagliflozinGruppe signifikant seltener als in der Placebo-Gruppe auf. Der Vorteil von Empagliflozin gegenüber Placebo hinsichtlich Neuauftreten oder Verschlechterung einer Nephropathie war konsistent in den untersuchten Subgruppen vorhanden. Die eGFR blieb unter Empagliflozin nach einer anfänglichen Abnahme über den weiteren Studienverlauf weitgehend konstant, während sie unter Placebo kontinuierlich abnahm ( $\mathbf{A b b}$.4). Die initiale eGFR-Abnahme unter Empagliflozin war nach Absetzen der Medikation bei Studienende weitgehend reversibel [31].

Die getrennte Auswertung für Empagliflozin $10 \mathrm{mg}$ und $25 \mathrm{mg}$ zeigte keine relevanten Unterschiede zwischen beiden Dosierungen für MACE-3, MACE-4, kardiovaskulär bedingten Tod, Gesamtmortalität, Hospitalisierungen wegen Herzinsuffizienz und neue oder sich verschlechternde Nephropathie [22, 30, 31].

\section{Sicherheit}

Insgesamt war der Anteil der Patienten mit unerwünschten Ereignissen (UE), schwerwiegenden UE und UE, die zum Absetzen der Studienmedikation führten, in den beiden Empagliflozin-Gruppen und der Placebo-Gruppe vergleichbar ( Tab. 2) [22]. Auf den Wirkmechanismus von SGLT-2-Inhibitoren zurückzuführende Genitalinfektionen traten in den Empagliflozin-Gruppen häufiger auf. Die diabetische Ketoazidose war in allen Behandlungsgruppen vergleichbar selten (Anteil der betroffenen Patienten: Empagliflozin $10 \mathrm{mg}$ [0,1\%] und $25 \mathrm{mg}[<0,1 \%]$, Placebo [<0,1\%]). Die Häufigkeit bestätigter Hypoglykämien war ebenfalls zwischen den Studienarmen vergleichbar: Empagliflozin $10 \mathrm{mg}$ (28\%) und $25 \mathrm{mg}$ (27,6\%), Placebo (27,9\%). Auch die Zahl der schweren Hypoglykämien mit Notwendigkeit zur Fremdhilfe war unter Placebo und beiden Empagliflozin-Dosierungen vergleichbar 
- Tab. 2 Unerwünschte Ereignisse.

\begin{tabular}{|c|c|c|c|}
\hline Ereignis & $\begin{array}{l}\text { Placebo } \\
(\mathrm{N}=2333)\end{array}$ & $\begin{array}{l}\text { Empagliflozin } 10 \mathrm{mg} \\
\qquad(\mathrm{N}=2345)\end{array}$ & $\begin{array}{l}\text { Empagliflozin } 25 \mathrm{mg} \\
\qquad(\mathrm{N}=2342)\end{array}$ \\
\hline UE insgesamt & $2139(91,7 \%)$ & $2112(90,1 \%)$ & $2118(90,4 \%)$ \\
\hline UE mit schwerer Ausprägung & $592(25,4 \%)$ & $536(22,9 \%)$ & $564(24,1 \%)$ \\
\hline \multicolumn{4}{|l|}{ Schwerwiegendes UE } \\
\hline - Insgesamt & $988(42,3 \%)$ & $876(37,4 \%)$ & $913(39,0 \%)$ \\
\hline - Tod & $119(5,1 \%)$ & $97(4,1 \%)$ & $79(3,4 \%)$ \\
\hline $\begin{array}{l}\text { Zum Abbruch der Einnahme von Studienmedikation } \\
\text { führendes UE }\end{array}$ & $453(19,4 \%)$ & $416(17,7 \%)$ & $397(17,0 \%)$ \\
\hline \multicolumn{4}{|l|}{ Bestätigte Hypoglykämie ${ }^{1}$} \\
\hline - Insgesamt & $650(27,9 \%)$ & $656(28,0 \%)$ & $647(27,6 \%)$ \\
\hline - Intervention notwendig & $36(1,5 \%)$ & $33(1,4 \%)$ & $30(1,3 \%)$ \\
\hline Harnwegsinfektion & $423(18,1 \%)$ & $426(18,2 \%)$ & $416(17,8 \%)$ \\
\hline - Männer & $158(9,4 \%)$ & $180(10,9 \%)$ & $170(10,1 \%)$ \\
\hline - Frauen & $265(40,6 \%)$ & $246(35,5 \%)$ & $246(37,3 \%)$ \\
\hline Komplizierte Harnwegsinfektion & $41(1,8 \%)$ & $34(1,4 \%)$ & $48(2,0 \%)$ \\
\hline Genitalinfektion & $42(1,8 \%)$ & $153(6,5 \%)$ & $148(6,3 \%)$ \\
\hline - Männer & $25(1,5 \%)$ & $89(5,4 \%)$ & $77(4,6 \%)$ \\
\hline - Frauen & $17(2,6 \%)$ & $64(9,2 \%)$ & $71(10,8 \%)$ \\
\hline Volumenmangel & $115(4,9 \%)$ & $115(4,9 \%)$ & $124(5,3 \%)$ \\
\hline Akutes Nierenversagen & $155(6,6 \%)$ & $121(5,2 \%)$ & $125(5,3 \%)$ \\
\hline Akuter Nierenschaden & $37(1,6 \%)$ & $26(1,1 \%)$ & $19(0,8 \%) \#$ \\
\hline Diabetische Ketoazidose & $1(<0,1 \%)$ & $3(0,1 \%)$ & $1(<0,1 \%)$ \\
\hline Thromboembolisches Ereignis & $20(0,9 \%)$ & $9(0,4 \%)$ & $21(0,9 \%)$ \\
\hline Knochenfraktur & $91(3,9 \%)$ & $92(3,9 \%)$ & $87(3,7 \%)$ \\
\hline
\end{tabular}

( $\triangleright$ Tab. 2). In den Empagliflozin-Gruppen wurden weniger Patienten mit akutem Nierenversagen oder akuten Nierenschäden dokumentiert als in der Placebo-Gruppe [22].

\section{Diskussion}

\section{Studien zur Beurteilung der kardiovaskulären Sicherheit}

In einer Reihe von Studien, über die \ Tab. 3 einen Überblick gibt, wurde die kardiovaskuläre Sicherheit neuer Antidiabetika im Vergleich zu Placebo untersucht. Diese Studien unterscheiden sich von früheren darin, dass die Patienten zusätzlich zur Studienmedikation mit einer optimierten antidiabetischen und kardiovaskulären (Antihypertensiva, Lipidsenker und Thrombozytenaggregationshemmer) Standardtherapie behandelt wurden, die im Studienverlauf angepasst werden konnte. Der kombinierte primäre Endpunkt war die Zeit bis zum ersten Auftreten von kardiovaskulärem Tod, nicht tödlichem Myokardinfarkt oder nicht tödlichem Schlaganfall (MACE-3) bei SAVOR-TIMI 53, EXAMINE,
LEADER, SUSTAIN-6 und EMPA-REG OUTCOME ${ }^{\circledR}$, bzw. MACE-3 plus Hospitalisierung wegen instabiler Angina pectoris (MACE-4) bei TECOS und ELIXA [22 - 24, 32 - 35]. Untersucht wurden neuere Antidiabetika der folgenden Klassen: Dipeptidylpeptidase4(DPP-4)-Inhibitoren (SAVOR-TIMI 53, EXAMINE, TECOS), GLP-1Rezeptoragonisten (ELIXA, LEADER, SUSTAIN-6) und SGLT-2-Inhibitoren (Empagliflozin). In der SAVOR-TIMI-53-Studie führte die Therapie mit Saxagliptin weder zu einem Anstieg noch zu einer Abnahme kardiovaskulärer Ereignisse, allerdings war die Rate von Hospitalisierungen aufgrund von Herzinsuffizienz erhöht [32]. Auch in den Studien EXAMINE, TECOS und ELIXA wurden keine Unterschiede im Auftreten kardiovaskulärer Ereignisse zwischen der jeweiligen Verumgruppe und Placebo beobachtet [33-35]. Damit konnten sie den von der US-amerikanischen Arzneimittelbehörde FDA geforderten Nachweis der kardiovaskulären Sicherheit erbringen. 
- Tab. 3 Übersicht abgeschlossener kardiovaskulärer Endpunktstudien mit neuen Antidiabetika gemäß regulatorischen Vorgaben seit 2008.

\begin{tabular}{|c|c|c|c|c|c|c|}
\hline Studie & Patienten & N & Intervention & $\begin{array}{l}\text { Mediane Follow- } \\
\text { up-Dauer }\end{array}$ & $\begin{array}{l}\text { Primärer } \\
\text { Endpunkt }\end{array}$ & $\begin{array}{c}\text { HR } \\
(95 \% \mathrm{KI})\end{array}$ \\
\hline SAVOR-TIMI 53 (20) & $\begin{array}{l}\text { T2DM+CVD/CRF } \\
\mathrm{HbA}_{1 \mathrm{c}} 6,5-12,0 \%\end{array}$ & 16492 & Saxagliptin vs. Placebo & 2,1 Jahre & MACE-3 & $\begin{array}{c}1,00 \\
(0,89-1,12)\end{array}$ \\
\hline EXAMINE (21) & $\begin{array}{l}\text { T2DM+ACS } \\
\mathrm{HbA}_{1 \mathrm{c}} 6,5-11,0 \%\end{array}$ & 5380 & Alogliptin vs. Placebo & 1,5 Jahre & MACE-3 & $\begin{array}{c}0,96 \\
\left(1,16^{1}\right)\end{array}$ \\
\hline $\operatorname{TECOS}(22)$ & $\begin{array}{l}\text { T2DM+CVD } \\
\mathrm{HbA}_{1 \mathrm{c}} 6,5-8,0 \%\end{array}$ & 14671 & Sitagliptin vs. Placebo & 3,0 Jahre & MACE-4 & $\begin{array}{c}0,98 \\
(0,88-1,09)\end{array}$ \\
\hline ELIXA (23) & $\begin{array}{l}\text { T2DM+ACS } \\
\mathrm{HbA}_{1 \mathrm{c}} 5,5-11 \%\end{array}$ & 6068 & Lixisenatid vs. Placebo & 2 Jahre & MACE-4 & $\begin{array}{c}1,02 \\
0,89-1,17\end{array}$ \\
\hline LEADER (24) & $\begin{array}{l}\mathrm{T} 2 \mathrm{DM}+\mathrm{CVD} / \mathrm{CRF} \\
\mathrm{HbA}_{1 \mathrm{c}} \geq 7,0 \%\end{array}$ & 9340 & Liraglutid vs. Placebo & 3,8 Jahre & MACE-3 & $\begin{array}{c}0,87 \\
(0,78-0,97)\end{array}$ \\
\hline SUSTAIN-6 $(25)^{2}$ & $\begin{array}{l}\text { T2DM+CVD/CRF } \\
\mathrm{HbA}_{1 \mathrm{c}} \geq 7,0 \%\end{array}$ & 3297 & Semaglutid vs. Placebo & 2,1 Jahre & MACE-3 & $\begin{array}{c}0,74 \\
(0,58-0,95)\end{array}$ \\
\hline $\begin{array}{l}\text { EMPA-REG OUTCOME } \\
\text { (27) }\end{array}$ & $\begin{array}{l}\text { T2DM+CVD } \\
\mathrm{HbA}_{1 \mathrm{c}} 7,0-10 \%\end{array}$ & 7020 & Empagliflozin vs. Placebo & 3,1 Jahre & MACE-3 & $\begin{array}{c}0,86 \\
(0,74-0,99)\end{array}$ \\
\hline
\end{tabular}

ACS: Akutes Koronarsyndrom; CRF: kardiovaskuläre Risikofaktoren; CVD: kardiovaskuläre Erkrankung; HR: Hazard Ratio; KI: Konfidenzintervall; MACE-3: Zeit bis zum Auftreten von kardiovaskulärem Tod, nicht tödlichem Myokardinfarkt oder nicht tödlichem Schlaganfall; MACE-4: MACE-3 + Hospitalisierung wegen instabiler Angina pectoris; T2DM: Typ-2-Diabetes mellitus.

1 oberes Konfidenzintervall.

2 Phase-3-Zulassungsstudie, Testung auf Überlegenheit war nicht präspezifiziert.

\section{Gegenüberstellung der Ergebnisse von kardiovasku- lären Endpunktstudien mit neueren Antidiabetika}

Mit Empagliflozin wurde erstmals in einer großen Endpunktstudie eine signifikante Senkung des relativen Risikos für den präspezifizierten primären Endpunkt MACE-3, Gesamtmortalität, Hospitalisierung aufgrund von Herzinsuffizienz und renale Endpunkte bei Patienten mit T2DM und bestehender kardiovaskulärer Erkrankung gezeigt [22, 30, 31]. Für eine über drei Jahre behandelte Gesamtpopulation wies Empagliflozin eine Number Needed to Treat (NNT) von 39 auf, sodass pro 39 über drei Jahre mit Empagliflozin behandelte Patienten ein Todesfall verhindert werden konnte [22]. Auch in den später publizierten LEADER- und SUSTAIN-6Studien konnte ein Vorteil der GLP-1-Rezeptoragonisten Liraglutid und Semaglutid gegenüber Placebo für MACE-3 und renale Endpunkte demonstriert werden. Für Liraglutid und Semaglutid konnte jedoch keine signifikante Risikoreduktion für stationäre Aufnahmen wegen Herzinsuffizienz beobachtet werden; bei Semaglutid wurde zudem keine Risikoreduktion der Gesamtmortalität festgestellt ( $\vee$ Tab. 4; $[23,24]$ ). Aus der LEADER-Studie ergibt sich für eine dreijährige Liraglutid-Behandlung eine NNT von 98 zur Vermeidung eines Todesfalls [23]. Als grundlegender Unterschied im Design dieser drei Studien ist zu beachten, dass in SUSTAIN-6 im Gegensatz zu EMPA-REG OUTCOME ${ }^{\circledR}$ und LEADER die Testung auf Überlegenheit im primären Endpunkt nicht präspezifiziert war und es sich um eine vor der Zulassung abgeschlossene Phase-3-Studie handelte. Ein weiterer Unterschied zwischen den Studien EMPA-REG OUTCOME ${ }^{\circledR}$, LEADER und SUSTAIN-6 besteht im Hinblick auf das Vorhandensein kardiovaskulärer Risikofaktoren, kardiovaskulärer Vorerkrankung (z. B. akutes Koronar- syndrom, Herzinsuffizienz) und chronischer Nierenerkrankung bei den eingeschlossenen Patienten ( $\downarrow$ Tab. $\mathbf{3})$. Darüber hinaus war die mediane Beobachtungszeit in SUSTAIN-6 mit 2,1 Jahren kürzer als bei EMPA-REG OUTCOME ${ }^{\circledR}$ mit 3,1 bzw. LEADER mit 3,8 Jahren ( $\triangleright$ Tab. 3 ).

\section{Gegenüberstellung der Ergebnisse weiterer kardiovaskulärer Endpunktstudien}

Bei allen anderen bislang abgeschlossenen Studien zur kardiovaskulären Sicherheit (SAVOR-TIMI 53, EXAMINE, TECOS, ELIXA) lag die Obergrenze des Konfidenzintervalls für den primären Endpunkt (MACE-3 bzw. MACE-4) zwar unterhalb des von der FDA für Nichtunterlegenheit geforderten Werts von 1,3, jedoch konnte in keiner dieser Studien eine signifikante Senkung des kardiovaskulären Risikos durch die untersuchten Substanzen beobachtet werden [32-35]. Andere blutzuckersenkende Therapeutika aus der Gruppe der Thiazolidindione (Glitazone) und der DDP-4-Inhibitor Saxagliptin wurden mit einem erhöhten Risiko für Hospitalisierung aufgrund von Herzinsuffizienz in Verbindung gebracht $[32,36]$. Im Vergleich zu den Studien SAVOR-TIMI 53, TECOS, EXAMINE, ELIXA, LEADER und SUSTAIN-6 zeigte EMPA-REG OUTCOME ${ }^{\circledR}$ die niedrigste auf das Jahr umgerechnete Rate an Krankenhauseinweisungen aufgrund von Herzinsuffizienz [22 - 24, 30, $32-35]$.

Für die Substanzklasse der Sulfonylharnstoffe ist die Datenlage hinsichtlich kardiovaskulärer Endpunkte widersprüchlich. So zeigte die UGDP-Studie Ende der neunziger Jahre für Tolbutamid gegenüber Placebo eine Erhöhung kardiovaskulärer Todesfälle, was zunächst zu Sicherheitsbedenken führte [37]. In UKPDS 33 und 


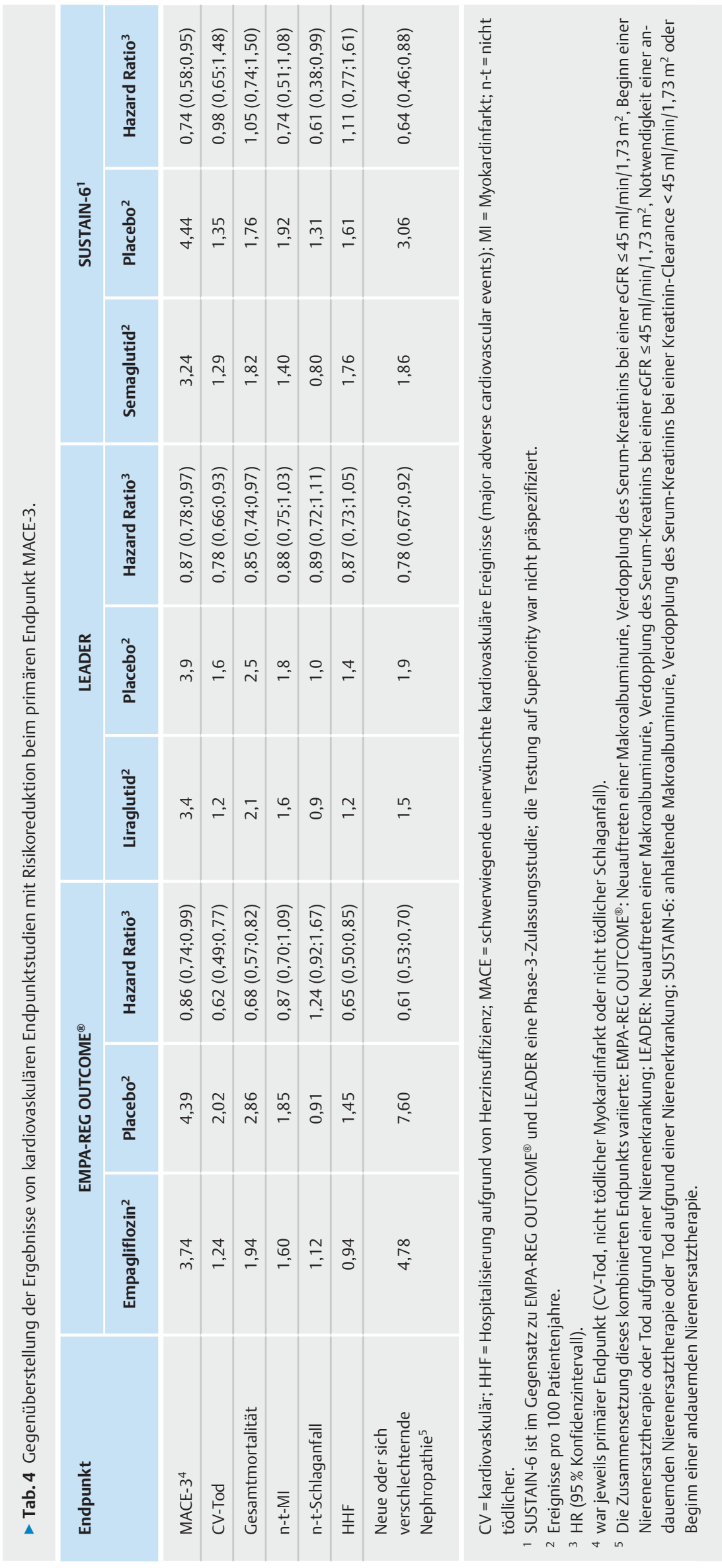


ADVANCE konnte später jedoch die kardiovaskuläre Sicherheit von Sulfonylharnstoffen gezeigt werden [38, 39], und auch eine Metaanalyse aus dem Jahr 2013 bestätigte unter Einschluss von 115 randomisierten klinischen Studien keine Erhöhung des Risikos für MACE von Sulfonylharnstoffen gegenüber Vergleichstherapien [40]. Auch in der Gruppe der Glitazone ist die Datenlage heterogen. Zwar fand sich für Muraglitazar im FDA-Assessment ein Anhalt für eine potenzielle Erhöhung des kardiovaskulären Risikos [41], und auch Rosiglitazon zeigte ein erhöhtes Risiko für Myokardinfarkt und kardiovaskulären Tod [20], wohingegen die Open-Label-Daten aus RECORD keine Erhöhung von kardiovaskulären Todesfällen unter Rosiglitazon nachweisen konnten [42] und PROactive für Pioglitazon sogar eine signifikante 16\%ige Reduktion von MACE-3 als sekundärem Endpunkt ergab [43].

UKPDS zeigte für Insuline bei intensiver glykämischer Kontrolle eine Verbesserung mikrovaskulärer, jedoch nicht makrovaskulärer Endpunkte [44], und erst im Langzeit Follow-up über 10 Jahre ergab sich ein makrovaskulärer Benefit im Sinne einer Reduktion von Myokardinfarkten unter intensivierter glykämischer Kontrolle [16]. Insbesondere für hypoglykämische Episoden werden ungünstige Auswirkungen und Zusammenhänge mit kardiovaskulären Risiken diskutiert [45].

Zwischen den aktuellen Studien EMPA-REG OUTCOME ${ }^{\circledR}$, LEADER und SUSTAIN-6, die allesamt eine signifikante Risikoreduktion im primären kombinierten Endpunkt MACE-3 zeigen konnten, bestanden allerdings Unterschiede im Beitrag der Einzelkomponenten. Im Gegensatz zu Liraglutid, bei dem in der LEADER-Studie alle drei MACE-Komponenten zu dem Ergebnis beitrugen, waren bei Empagliflozin in der EMPA-REG OUTCOME ${ }^{\circledR}$ Studie der kardiovaskuläre Tod mit einer RRR von $38 \%$ und bei Semaglutid in SUSTAIN-6 der nicht tödliche Schlaganfall mit einer RRR von $39 \%$ die Komponenten mit dem stärksten Effekt ( Tab. 4; [22 - 24]). In der EMPA-REG OUTCOME ${ }^{\circledR}$-Studie waren nicht tödliche Myokardinfarkte und Schlaganfälle nicht signifikant verändert [22, 46]. Auch hinsichtlich renaler Outcomes unterschieden sich diese Studien mit einem ausgeprägteren Benefit unter Empagliflozin (RRR 39\% in EMPA-REG OUTCOME ${ }^{\circledR}$ ) und Semaglutid (RRR $36 \%$ in SUSTAIN-6) als unter Liraglutid (RRR $22 \%$ in LEADER), wobei die genaue Definition des kombinierten Nephropathie-Endpunktes geringfügig zwischen den Studien variierte $(\triangleright$ Tab. 4; $[23,24,31])$.

Bei der EMPA-REG OUTCOME ${ }^{\circledR}$-Studie lässt die frühzeitige Trennung der Ereigniskurven bei den Endpunkten kardiovaskulärer Tod, Hospitalisierung aufgrund von Herzinsuffizienz und Gesamtmortalität zwischen Empagliflozin und Placebo ( $>$ Abb. 2b, 3a, b) einen wesentlichen Effekt eines nicht $\mathrm{HbA}_{1 c}$-basierten Mechanismus vermuten.

\section{Sicherheits- und Verträglichkeitsprofil von Empagli- flozin in der EMPA-REG OUTCOME ${ }^{\circledR}$-Studie}

In der EMPA-REG OUTCOME ${ }^{\circledR}$-Studie war der Anteil der Patienten mit UE, schwerwiegenden UE und UE, die zum Absetzen der Studienmedikation führten in allen Armen vergleichbar. Auch die Häufigkeit der unter SGLT-2-Inhibition auftretenden diabetischen Ketoazidose war in den beiden Empagliflozin-Gruppen und der Placebo-Gruppe vergleichbar ( $\vee$ Tab. 2). Eine umfassende Sicher- heitsanalyse von Empagliflozin zeigte anhand gepoolter Daten von über 12000 Patienten aus 17 Studien der Phasen I-III (zuzüglich 6 Verlängerungsstudien), die auf Placebo, Empagliflozin $10 \mathrm{mg}$ oder $25 \mathrm{mg}$ randomisiert waren, eine in allen drei Gruppen vergleichbare Gesamtinzidenz von UE, schwerwiegenden UE, schweren UE und UE, die zum Abbruch der Studienmedikation führten [47]. Empagliflozin war im Vergleich zu Placebo mit keiner höheren Inzidenz von Hypoglykämien assoziiert, außer in Kombination mit Sulfonylharnstoff oder Insulin. Die Häufigkeit von diabetischer Ketoazidose war in dieser Metaanalyse vergleichbar für Empagliflozin und Placebo (Anteil der betroffenen Patienten: Empagliflozin $10 \mathrm{mg}[0,1 \%]$ und $25 \mathrm{mg}[<0,1 \%]$, Placebo [0,1\%]) [47]. Die Ergebnisse zu den Sicherheitsendpunkten sind somit in dieser Metaanalyse und der EMPA-REG OUTCOME ${ }^{\circledR}$-Studie konsistent und bestätigen ein günstiges Risiko-Nutzen-Profil von Empagliflozin.

\section{Potenzielle Wirkmechanismen}

Über welchen Mechanismus bzw. welche Mechanismen die protektive Wirkung von Empagliflozin auf das kardiovaskuläre sowie renale Risiko vermittelt wird, ist bisher nicht geklärt. Generell führt Empagliflozin zur Blutzuckersenkung, indem es die renale Glukoserückresorption verringert und somit eine verstärkte Glukoseausscheidung über den Urin bewirkt. Darüber hinaus wurde mit Empagliflozin eine positive Wirkung auf Blutdruck, Gewicht, viszerale Adipositas, Hyperinsulinämie, arterielle Steifigkeit, Albuminurie, zirkulierende Harnsäurekonzentration und oxidativen Stress beobachtet [48]. Insbesondere die frühzeitige Risikoreduktion von kardiovaskulärem Tod, stationären Aufnahmen wegen Herzinsuffizienz sowie neuer oder sich verschlechternder Nephropathie nach Therapiebeginn deuten darauf hin, dass diese Effekte eher hämodynamischer Natur sind und nicht auf eine Vermeidung von Atherosklerose zurückführbar sein dürften [49]. Ebenfalls denkbar ist ein Zusammenspiel mehrerer Wirkmechanismen.

Die SGLT-2-Inhibition führt nicht nur zur Hemmung der Rückresorption von Glukose, sondern auch von Natrium. Es wird vermutet, dass dadurch eintretende renale hämodynamische Effekte für die Nephroprotektion verantwortlich sein könnten [50]. Infolge der erhöhten Glukosemenge im Primärharn kommt es bei Patienten mit Diabetes mellitus zu einer Hochregulation der SGLT-2Expression. Eine erhöhte Zahl von SGLT-2-Molekülen würde aufgrund des Co-Transports von Natrium und Glukose in einer verminderten Natriummenge an der Macula densa resultieren. Dies könnte über ein tubulo-glomeruläres Feedback eine Dilatation des glomerulären Vas afferens mit konsekutiver intraglomerulärer Druckerhöhung und renaler Hyperfiltration erklären. Diese Hyperfiltration entspräche auch den frühen Zeichen einer diabetischen Nephropathie und dieser könnte durch SGLT-2-Inhibition entgegengewirkt werden, da vermehrt Natrium zur Macula densa gelangen würde. Dies könnte über ein tubulo-glomeruläres Feedback die Verengung des glomerulären Vas afferens bewirken und würde damit zu einer Normalisierung des intraglomerulären Drucks und Reduktion der Hyperfiltration führen. Eine Abschwächung der renalen Hyperfiltration konnte bei Patienten mit Typ1-Diabetes gezeigt werden [51]. Die in der EMPA-REG OUTCO$\mathrm{ME}^{\circledR}$-Studie beobachtete langsamere Progression der Niereninsuf- 
fizienz und niedrigere Rate an Nephropathien in der Empagliflozin-Gruppe gegenüber Placebo ist von besonderer klinischer Bedeutung, da Diabetes mellitus die häufigste Ursache für die Entwicklung einer terminalen Niereninsuffizienz in vielen Teilen der Welt ist [10 - 12].

Die Kombination aus vermehrter Ausscheidung von Glukose, Natrium und Wasser sowie normalisiertem tubulo-glomerulären Feedback könnte den kardialen und renalen Benefit der SGLT-2Hemmung erklären: Indem der Flüssigkeitsverlust durch die Ausscheidung von Glukose und Natrium über den Urin erhöht wird, kommt es außerdem zur Senkung von intravaskulärem Volumen und systolischem Blutdruck sowie zu einer signifikanten Erhöhung des Hämatokrits. Diese Veränderungen reduzieren kardiale Stressfaktoren und verbessern möglicherweise zusätzlich die myokardiale Sauerstoffverfügbarkeit. Daraus ergäbe sich eine Entlastung des Herzens und somit eine Verbesserung der systolischen und diastolischen Funktion, eine Verringerung der Wahrscheinlichkeit für Lungenödeme und somit eine Risikoreduktion für Hospitalisierungen wegen Herzinsuffizienz und tödlich verlaufende Arrhythmien. Eine Verbesserung der kardialen Funktion könnte wiederum zu einer besseren Nierendurchblutung und Nierenfunktion führen [52].

Ein weiterer Erklärungsansatz für den kardio-renalen Nutzen liegt in einer günstigen Beeinflussung der kardialen Energienutzung, beispielsweise über Ketonkörper, die unter SGLT-2-Hemmung vermehrt auftreten können („Thrifty Substrate“- oder „Fuel“-Hypothese) [53-55].

\section{Übertragbarkeit der Ergebnisse der EMPA-REG OUTCOME ${ }^{\circledR}$-Studie}

Ob die in der EMPA-REG OUTCOME ${ }^{\circledR}$-Studie gewonnenen Erkenntnisse auf andere SGLT-2-Inhibitoren übertragbar sind, werden derzeit noch laufende Studien zu Dapagliflozin (DECLARE-TIMI58, NCT01 730 534), Canagliflozin (CREDENCE, NCT02 065 791) und Ertugliflozin (VERTIS, NCT01 986881 ) zeigen, deren Ergebnisse 2017 - 2020 erwartet werden. Eine Metaanalyse mit 11292 Patienten aus acht Phase-3-Studien weist darauf hin, dass auch bei Patienten mit geringem oder mittlerem kardiovaskulären Risiko Empagliflozin im Vergleich zu Placebo zu einer Risikoreduktion bei MACE-3 (HR 0,66; $95 \%$ KI: 0,39; 1,12) und MACE-4 (HR 0,59; $95 \%$ KI: 0,36; 0,95) führen könnte [57].

\section{Fazit}

Zusammenfassend steht mit Empagliflozin eine Therapieoption zur Verfügung, mit der das kardiovaskuläre Risiko von Patienten mit T2DM und kardiovaskulärer Vorerkrankung reduziert werden kann. Aufgrund der Ergebnisse aus der EMPA-REG OUTCOME ${ }^{\circledR}$ Studie wie auch anderer Antidiabetika mit positiven Ergebnissen aus kardiovaskulären Endpunktstudien ist ein erheblicher Einfluss auf die zukünftige Wahl der Therapiestrategien bei T2DM anzunehmen. Dies spiegelt sich bereits durch die Erwähnung von Empagliflozin in den Leitlinien der Deutschen Diabetes Gesellschaft (DDG), der Kanadischen (CDA) und Amerikanischen (ADA) Diabetes-Gesellschaften, der Amerikanischen Endokrinologie-Gesellschaften (AACE/ACE) wie auch in zwei Leitlinien der Europäischen Gesellschaft für Kardiologie (ESC) wider [58-63].
Aufgrund der vorliegenden Daten können vor dem Hintergrund des hohen kardiovaskulären Risikos und der potenziell schlechten Prognose für Niereninsuffizienz Patienten mit T2DM in vielfältiger Hinsicht von Empagliflozin profitieren, da die Substanz nicht nur das Risiko für kardiovaskulären Tod, Gesamtmortalität und Krankenhauseinweisungen wegen Herzinsuffizienz senkt, sondern sich auch positiv auf renale Endpunkte und die Erhaltung der glomerulären Filtrationsrate auswirkt. Die besondere Bedeutung der positiven Ergebnisse der EMPA-REG OUTCOME ${ }^{\circledR}$-Studie wird auch dadurch deutlich, dass Empagliflozin als erstes Antidiabetikum vom Gemeinsamen Bundesausschuss ( $G$-BA) einen beträchtlichen Zusatznutzen bei Typ-2-Diabetikern mit kardiovaskulärer Vorerkrankung in der oralen Zwei-, Drei- und MehrfachKombinationstherapie sowie der Add-on-Therapie mit Insulin zuerkannt bekam [64].

\section{Addendum}

Kürzlich wurden die Ergebnisse zu den gepoolten CANVAS-Studien, CANVAS und CANVAS-R, publiziert. Diese sich im Studiendesign, der Studienpopulation und den präspezifizierten Endpunkten von der EMPA-REG OUTCOME ${ }^{\circledR}$-Studie unterscheidende Studie zeigte für Canagliflozin eine Superiority für MACE-3. Hinsichtlich anderer Endpunkte gab es jedoch teils signifikant abweichende Ergebnisse im Vergleich zur EMPA-REG OUTCOME ${ }^{\circledR}$-Studie [65].

Interessenkonflikt

J. Seufert hat Vortragshonorare und/oder Beraterhonorare und/oder Forschungsförderung (als zweckgebundene Drittmittel) von Apitope, Astra Zeneca, Bayer, Berlin Chemie, Boehringer Ingelheim, Bristol Myers Squibb (BMS), GI-Dynamics, Glaxo Smith Kline (GSK), Intarcia, Ipsen, Janssen, LifeScan, Lilly, Merck Sharp Dohme (MSD), MedScape, Novartis, NovoNordisk, Omniamed, Pfizer, Roche, Sanofi Aventis, Servier, Takeda und Ypsomed erhalten.

J. Galle hat Honorare für Vortragstätigkeit und/oder Beratertätigkeit von AMGEN, Fresenius Medical Care (FMC), Hexal, Boehringer Ingelheim und Roche erhalten.

M. Manning ist Angestellte der Firma Lilly Deutschland GmbH, Medizinische Abteilung, und besitzt Aktien von Eli Lilly and Company. V. Schmid ist Angestellter der Firma Boehringer Ingelheim Pharma GmbH \& Co. KG, Medical Affairs Deutschland.

M. Lehrke hat Honorare für Referententätigkeit und Beratung von Boehringer Ingelheim, Astra Zeneca, Novo Nordisk, MSD, Sanofi-Aventis und AMGEN erhalten. Es besteht Forschungsunterstützung durch Boehringer Ingelheim.

\section{Danksagung}

Medical-Writing-Unterstützung erfolgte durch Petra Jöstingmeyer (med:unit GmbH, Köln).

Literatur

[1] Meigs JB. Epidemiology of cardiovascular complications in type 2 diabetes mellitus. Acta Diabetol 2003; 40 (Suppl. 2): S358 - S361

[2] Sarwar N, Gao P, Seshasai SR et al. Diabetes mellitus, fasting blood glucose concentration, and risk of vascular disease: a collaborative metaanalysis of 102 prospective studies. Lancet 2010; 375: 2215-2222 
[3] Shah AD, Langenberg C, Rapsomaniki E et al. Type 2 diabetes and incidence of cardiovascular diseases: a cohort study in 1.9 million people. Lancet Diabetes Endocrinol 2015; 3: 105-113

[4] Franco OH, Steyerberg EW, Hu FB et al. Associations of diabetes mellitus with total life expectancy and life expectancy with and without cardiovascular disease. Arch Intern Med 2007; 167: 1145-1151

[5] Go AS, Mozaffarian D, Roger VL et al. Heart disease and stroke statistics2013 update: a report from the American Heart Association. Circulation 2013; 127 (1): e6 - e245

[6] Bundesärztekammer (BÄK), Kassenärztliche Bundesvereinigung (KBV), Arbeitsgemeinschaft der Wissenschaftlichen Medizinischen Fachgesellschaften (AWMF). Nationale VersorgungsLeitlinie Therapie des Typ-2Diabetes - Langfassung. 2013. zuletzt geändert: November 2014. Verfügbar unter: 1. Auflage. Version 3: http://www.versorgungsleitlinien. de/themen/diabetes2/dm2_therapie

[7] American Diabetes Association. Cardiovascular Disease and Risk Management. Diabetes Care 2016; 39 (Suppl. 1): S60-S71

[8] UK Prospective Diabetes Study (UKPDS) Group. Intensive blood-glucose control with sulphonylureas or insulin compared with conventional treatment and risk of complications in patients with type 2 diabetes (UKPDS 33). Lancet 1998; 352: $837-853$

[9] Hemmingsen B, Lund SS, Gluud C et al. Targeting intensive glycaemic control versus targeting conventional glycaemic control for type $2 \mathrm{di}$ abetes mellitus. Cochrane Database Syst Rev 2013; 11: CD008143

[10] Toth-Manikowski S, Atta MG. Diabetic Kidney Disease: Pathophysiology and Therapeutic Targets. J Diabetes Res 2015; 2015: Article ID 697010

[11] Stewart JH, McCredie MR, Williams SM et al. Trends in incidence of treated end-stage renal disease, overall and by primary renal disease, in persons aged 20-64 years in Europe, Canada and the Asia-Pacific region, 1998-2002. Nephrology (Carlton) 2007; 12 (5): 520-527

[12] Thomas MC, Cooper ME, Zimmet P. Changing epidemiology of type 2 diabetes mellitus and associated chronic kidney disease. Nat Rev Nephrol 2016; 12: $73-81$

[13] National Kidney Foundation. 2015. Verfügbar unter: www.kidney.org/ atoz/content/diabetes

[14] Gregg EW, Li Y, Wang J et al. Changes in diabetes-related complications in the United States, 1990-2010. N Engl J Med 2014; 370: 1514-1523

[15] Hayward RA, Reaven PD, Wiitala WL et al. Follow-up of Glycemic Control and Cardiovascular Outcomes in Type 2 Diabetes. N Engl J Med 2015; 372: $2197-2206$

[16] Holman RR, Paul SK, Bethel MA et al. 10-year follow-up of intensive glucose control in type 2 diabetes. N Engl J Med 2008; 359: 1577 - 1589

[17] Gerstein HC, Miller ME, Genuth $S$ et al. Long-term effects of intensive glucose lowering on cardiovascular outcomes. N Engl J Med 2011; 364 : $818-828$

[18] Patel A, MacMahon S, Chalmers J et al. Intensive blood glucose control and vascular outcomes in patients with type 2 diabetes. $N$ Engl J Med 2008; 358: $2560-2572$

[19] Gerstein HC, Miller ME, Byington RP et al. Effects of Intensive Glucose Lowering in Type 2 Diabetes. N Engl J Med 2008; 358: 2545-2559

[20] Nissen SE, Wolski K. Effect of rosiglitazone on the risk of myocardial infarction and death from cardiovascular causes. N Engl J Med 2007; 356: $2457-2471$

[21] US Department of Health and Human Services, Food and Drug Administration, Center for Drug Evaluation and Research (CDER). Guidance for industry: diabetes mellitus - evaluating cardiovascular risk in new antidiabetic therapies to treat type 2 diabetes. 2008. Verfügbar unter: http://www.fda.gov/downloads/drugs/guidancecomplianceregulatoryinformation/guidances/ucm071627.pdf

[22] Zinman B, Wanner C, Lachin JM et al. Empagliflozin, Cardiovascular Outcomes, and Mortality in Type 2 Diabetes. N Engl J Med 2015; 373: 2117 2128
[23] Marso SP, Daniels GH, Brown-Frandsen K et al. Liraglutide and Cardiovascular Outcomes in Type 2 Diabetes. N Engl J Med 2016; 375: 311 322

[24] Marso SP, Bain SC, Consoli A et al. Semaglutide and Cardiovascular Outcomes in Patients with Type 2 Diabetes. N Engl J Med 2016; 375 : $1834-1844$

[25] Gallwitz B, Merker L, Hohberg C et al. Empagliflozin - Insulinunabhängige Kontrolle der Glykämieparameter bei Diabetes mellitus Typ 2 durch Inhibition des Natrium-Glukose-Cotransporters SGLT2. Diabetologie und Stoffwechsel 2015; 10: $247-265$

[26] Zinman B, Inzucchi SE, Lachin JM et al. Rationale, design, and baseline characteristics of a randomized, placebo-controlled cardiovascular outcome trial of empagliflozin (EMPA-REG OUTCOMETM). Cardiovasc Diabetol 2014; 13: 102

[27] Häring HU, Merker L, Seewaldt-Becker E et al. Empagliflozin as add-on to metformin in patients with type 2 diabetes: a 24-week, randomized, double-blind, placebo-controlled trial. Diabetes Care 2014; 37: 1650 1659

[28] Roden M, Weng J, Eilbracht J et al. Empagliflozin monotherapy with sitagliptin as an active comparator in patients with type 2 diabetes: a randomised, double-blind, placebo-controlled, phase 3 trial. Lancet Diabetes Endocrinol 2013; 1: 208-219

[29] Rosenstock J, Jelaska A, Frappin G et al. Improved glucose control with weight loss, lower insulin doses, and no increased hypoglycemia with empagliflozin added to titrated multiple daily injections of insulin in obese inadequately controlled type 2 diabetes. Diabetes Care 2014; 37 : $1815-1823$

[30] Fitchett D, Zinman B, Wanner C et al. Heart failure outcomes with empagliflozin in patients with type 2 diabetes at high cardiovascular risk: results of the EMPA-REG OUTCOME ${ }^{\circledR}$ trial. Eur Heart J 2016; 37 : 1526 1534

[31] Wanner C, Inzucchi SE, Lachin JM et al. Empagliflozin and Progression of Kidney Disease in Type 2 Diabetes. N Engl J Med 2016; 375: 323 - 334

[32] Scirica BM, Bhatt DL, Braunwald E et al. Saxagliptin and cardiovascular outcomes in patients with type 2 diabetes mellitus. N Engl J Med 2013; 369: $1317-1326$

[33] White WB, Cannon CP, Heller SR et al. Alogliptin after acute coronary syndrome in patients with type 2 diabetes. N Engl J Med 2013; 369: $1327-1335$

[34] Green JB, Bethel MA, Armstrong PW et al. Effect of Sitagliptin on Cardiovascular Outcomes in Type 2 Diabetes. N Engl J Med 2015; 373 : 232 242

[35] Pfeffer MA, Claggett B, Diaz R et al. Lixisenatide in Patients with Type 2 Diabetes and Acute Coronary Syndrome. N Engl J Med 2016; 373: $2247-2257$

[36] McMurray J], Gerstein HC, Holman RR et al. Heart failure: a cardiovascular outcome in diabetes that can no longer be ignored. Lancet Diabetes Endocrinol 2014; 2: $843-851$

[37] Meinert CL, Knatterud GL, Prout TE et al. A study of the effects of hypoglycemic agents on vascular complications in patients with adult-onset diabetes. II. Mortality results. Diabetes 1970; 19: 789-830

[38] UKPDS Group. Intensive blood-glucose control with sulphonylureas or insulin compared with conventional treatment and risk of complications in patients with type 2 diabetes (UKPDS 33). Lancet 1998; 352: 837 853

[39] Patel A, MacMahon S, Chalmers ] et al. Intensive blood glucose control and vascular outcomes in patients with type 2 diabetes. $N$ Engl J Med 2008; 358: $2560-2572$

[40] Monami M, Genovese S, Mannucci E. Cardiovascular safety of sulfonylureas: a meta-analysis of randomized clinical trials. Diabetes Obes Metab 2013; 15: $938-953$ 
[41] Nissen SE, Wolski K, Topol EJ. Effect of muraglitazar on death and major adverse cardiovascular events in patients with type 2 diabetes mellitus. JAMA 2005; 294: $2581-2586$

[42] Home PD, Pocock S], Beck-Nielsen H. Rosiglitazone evaluated for cardiovascular outcomes in oral agent combination therapy for type 2 diabetes (RECORD): a multicentre, randomised, open-label trial. Lancet 2009; 373: 2125-2135

[43] Dormandy JA, Charbonnel B, Eckland D] et al. Secondary prevention of macrovascular events in patients with type 2 diabetes in the PROactive Study (PROspective pioglitAzone Clinical Trial In macroVascular Events): a randomised controlled trial. Lancet 2005; 66: 1279-1289

[44] UKPDS Group. Intensive blood-glucose control with sulphonylureas or insulin compared with conventional treatment and risk of complications in patients with type 2 diabetes (UKPDS 33). Lancet 1998; 352: 837 853

[45] Khunti K, Davies M, Maieed A et al. Hypoglycemia and risk of cardiovascular disease and all-cause mortality in insulin-treated people with type 1 and type 2 diabetes: a cohort study. Diabetes Care 2015; 38: 316- 322

[46] Zinman B, Inzucchi SE, Lachin JM et al. Empagliflozin and Cerebrovascular Events in Patients With Type 2 Diabetes Mellitus at High Cardiovascular Risk Stroke; 2017; 48: 1218-1225

[47] Kohler S, Salsali A, Hantel S et al. Safety and Tolerability of Empagliflozin in Patients with Type 2 Diabetes. Clin Ther 2016; 38: 1299-1313

[48] Inzucchi SE, Zinman B, Wanner C et al. SGLT-2 inhibitors and cardiovascular risk: proposed pathways and review of ongoing outcome trials. Diab Vasc Dis Res 2015; 12: 90-100

[49] Kahles F, Marx N. Aktuelle kardiovaskuläre Outcome-Studien bei Diabetes. Diabetologe 2016; 12: 88-95

[50] Rajasekeran H, Lytvyn Y, Cherney DZ. Sodium-glucose cotransporter 2 inhibition and cardiovascular risk reduction in patients with type 2 diabetes: the emerging role of natriuresis. Kidney Int 2016; 89: 524- 526

[51] Cherney DZI, Perkins BA, Soleymanlou N et al. Renal Hemodynamic Effect of Sodium-Glucose Cotransporter 2 Inhibition in Patients With Type 1 Diabetes Mellitus. Circulation 2014; 129: 587-597

[52] Sattar N, McLaren J, Kristensen SL et al. SGLT2 Inhibition and cardiovascular events: why did EMPA-REG Outcomes surprise and what were the likely mechanisms? Diabetologia 2016; 59: 1333-1339

[53] Heerspink H, Perkins B, Fitchett D et al. SGLT2 inhibitors in the treatment of diabetes: Cardiovascular and kidney effects, potential mechanisms and clinical applications. Circulation 2016; 134: 752 - 772
[54] Mudaliar S, Alloju S, Henry R. Can a shift in fuel energetics explain the beneficial cardiorenal outcomes in the EMPA-REG OUTCOME study? A unifying hypothesis. Diabetes Care 2016; 39: 1115-1122

[55] Ferranini E, Mark M, Mayoux E. Protection in the EMPA-REG OUTCOMETM trial: a „thrifty substrate” hypothesis. Diabetes Care 2016; 39: 1108 1114

[56] Neal B, Perkovic V, de Zeeuw D et al. Rationale, design, and baseline characteristics of the Canagliflozin Cardiovascular Assessment Study (CANVAS)-a randomized placebo-controlled trial. Am Heart J 2013; 166 : 217-223.e11

[57] Salsali A, Kim G, Woerle H] et al. Cardiovascular safety of empagliflozin in patients with type 2 diabetes: a meta-analysis of data from randomized placebo-controlled trials. Diabetes Obes Metabol 2016; 18: 1034-1040

[58] Landgraf R, Kellerer M, Fach E et al. Praxisempfehlungen DDG/DGIM Therapie des Typ-2-Diabetes. Diabetologie und Stoffwechsel 2015; 11 (Suppl. 2): $140-151$

[59] Goldenberg R, Clement M, Hanna A et al. Policies, Guidelines and Consensus Statements - Pharmacologic Management of Type 2 Diabetes: 2016 Interim Update. Can J Diabetes 2016; 40: 193-195

[60] American Diabetes Association. Standards of Medical Care in Diabetes Position Statements. Diabetes Care 2016; 39 (Suppl. 1): S52 - S71

[61] Garber AJ, Abrahamson M], Barzilay Jl et al. Consensus Statement by the American Association of Clinical Endocrinologists and American College of Endocrinology on the comprehensive Type 2 Diabetes Management Algorithm - 2016 Executive Summary. Endocr Pract 2016; 22: 84-113

[62] Piepoli MF, Hoes AW, Agewall S et al. 2016 European Guidelines on cardiovascular disease prevention in clinical practice. Eur Heart J 2016; 37 : 2315-2381

[63] Ponikowski P, Voors AA, Anker SD et al. 2016 ESC Guidelines for the diagnosis and treatment of acute and chronic heart failure. Eur J Heart Fail 2016; 18: $891-975$

[64] Gemeinsamer Bundesausschuss. Tragende Gründe zum Beschluss des Gemeinsamen Bundesausschusses über eine Änderung der Arzneimittel-Richtlinie (AM-RL): Anlage XII - Beschlüsse über die Nutzenbewertung von Arzneimitteln mit neuen Wirkstoffen nach § 35a SGB V - Empagliflozin. 2016. Verfügbar unter: https://www.g-ba.de/downloads/ 40-268-3955/2016-09-01_AM-RL-XII_Empagliflozin_D-214_TrG.pdf

[65] Neal B, Perkovic V, Mahaffey KW et al. Canagliflozin and Cardiovascular and Renal Events in Type 2 Diabetes. N Engl J Med 2017. DOI: 10.1056/ NEJMoa1611925 